\title{
Depression and helplessness impact interstitial cystitis/bladder pain syndrome pain over time
}

Alison Crawford, $\mathrm{MSc}^{1}$; Dean A. Tripp, $\mathrm{PhD}^{2}$; J. Curtis Nickel, $\mathrm{MD}^{3}$; Lesley Carr, $\mathrm{MD}^{4}$; Robert Moldwin, $\mathrm{MD}^{5}$; Laura Katz, $\mathrm{PhD}, \mathrm{CPsych}^{6}$; Abi Muere, $\mathrm{MSc}^{1}$

${ }^{1}$ Department of Psychology, Queen's University, Kingston, ON, Canada; ${ }^{2}$ Departments of Psychology, Urology, \& Anesthesiology, Queen's University, Kingston, ON, Canada; ${ }^{3}$ Department of Urology Queen’s University, Kingston, ON, Canada; ${ }^{4}$ Department of Surgery, University of Toronto, Toronto, ON, Canada; ${ }^{5}$ Department of Urology, Hosftra University School of Medicine, New Hyde Park, NY, United States; ${ }^{6}$ Michael G. DeGroote Pain Clinic, McMaster University Hospital, Hamilton, ON, Canada

Cite as: Can Urol Assoc J 2019 February 7; Epub ahead of print. http://dx.doi.org/10.5489/cuaj.5703

Published online February 7, 2019

$* * *$

\section{Abstract}

Introduction: Interstitial cystitis/bladder pain syndrome (IC/BPS) is a devastating urological chronic pelvic pain condition with an unknown etiology. Evidence-based psychological strategies are becoming more successful for symptom management as we learn more about the targets for intervention. Previous research has established an indirect relationship between depression and pain through catastrophizing, but there have yet to be studies examining the emerging role of emotion regulation in this relationship.

Methods: Women with IC/BPS were recruited from tertiary care clinics in Canada and the U.S. between 2013 and 2018. Patients completed questionnaires, including demographics and scores for pain, depression, catastrophizing, and difficulties in emotion regulation at baseline, six months, and one year. Serial mediation was used to test models of pain, catastrophizing, and depression.

Results: A total of 135 women with IC/BPS completed all three time points. The only significant indirect path was from baseline depression to catastrophizing at six months, to pain at one year ( $\mathrm{b}=0.10$; confidence interval [CI] 0.0049-0.2520). A followup analysis demonstrated that helplessness was the key factor of catastrophizing driving this relationship ( $b=0.17$; CI 0.02820.3826).

Conclusions: Reducing feelings of helplessness and increasing patient feelings of control are important ways to limit the effect of low mood on patient pain experience. De-catastrophizing interventions should be part of the referral strategy for IC/BPS symptom management. 


\section{Introduction}

Interstitial Cystitis/Bladder Pain Syndrome (IC/BPS) is a chronic pelvic pain condition characterized by persistent pain as well as urinary urgency and frequency that has no cure or agreed upon etiology. ${ }^{1}$ Only a minority of patients report being symptom free at a one-year follow-up, ${ }^{2}$ and diagnoses of depression and anxiety are more common in women with IC/BPS. ${ }^{3}$

Research recommends a symptom management approach to IC/BPS based on the frequent and robust associations between psychosocial variables (i.e., depression and pain catastrophizing) and pain-associated outcomes. ${ }^{1,4,5}$ Pain catastrophizing is a negative mental bias that promotes greater pain sensitivity. ${ }^{6}$ Depression can further exacerbate pain, ${ }^{7}$ and is considered a catalyst to pain associated complications. ${ }^{8}$ Recently, depression assessed at baseline was shown to lead to worse outcomes in pain, but it is unclear whether baseline pain affects future depression, and what variables might mediate the relationship between pain and depression. ${ }^{9}$ In patients with IC/BPS, catastrophizing and depression have been found to be correlated with IC/BPS symptoms, and decreased physical and mental quality of life. ${ }^{10-12}$

Many biopsychosocial-based interventions also consider emotion self-regulation an essential target for change, but to date it is unexamined in urologic chronic pelvic pain. ${ }^{13}$ Emotion regulation is an important addition to understanding the pain experience because the strategies most often used to regulate emotions are similar to pain management strategies (e.g., distraction, cognitive reappraisal, avoidance, self-reward) ${ }^{14}$ and maladaptive emotion regulation is suggested as factor in the development and maintenance of chronic pain. ${ }^{13}$ Specifically, maladaptive emotion regulation has been found to predict the decrease of psychological and physical health over time. ${ }^{15}$

Models proposing how the depression to pain pathway may function have not been examined in IC/BPS or have been tested longitudinally. This study examined the longitudinal role of difficulties in emotion regulation in the indirect effect of catastrophizing between depression and pain. A particular focus was to examine the relationship from baseline depression to catastrophizing at six months, to difficulties in emotion regulation at six months, to pain at one-year. An alternate model from baseline pain to catastrophizing at six months, to difficulties in emotion regulation at six months, to depression at one-year was also examined

\section{Method}

\section{Participants}

Women diagnosed with IC/BPS were drawn from three tertiary care clinics in Canada and the United States $(N=226)$. These clinics were located in Kingston, ON, Toronto ON, and New York from dates of study. Informed consent was obtained from these women, and this study was cleared by the Hospital Research Ethics Board at Queen's University. Data were collected between 2013 and 2018. Women were included in this study if they completed the questionnaire at all three time points: baseline, six months, and one year $(N=135)$. All participants were 
female, have a Urologist diagnosis of IC/BPS, able to read and write in English, and were 18 years of age or older. The mean age of the participants who completed the study was 52.57 years $(S D=15.51)$, and the average time since their diagnosis was 8.67 years $(S D=9.11)$, with $17.04 \%(n=23)$ having had their diagnosis for one year or less. The demographic characteristics of the sample are outlined in Table 1. The sample was predominantly white, college/university educated, living with a spouse/partner, and employed. The types of treatments the patients received throughout and during the study are outlined in Table 2. Most patients received medication related interventions. Surgical interventions, physical manipulations, psychological interventions and a number of alternative treatments were also common.

\section{Procedure}

A standardized REB approved protocol was used across sites and institutional clearance was obtained. Participants were approached individually either before or after their Urology Outpatient clinical appointments and were provided basic information about the study by a research nurse. Those patients who were interested in participating were then briefed about the study. If they indicated interest, they were sent home with a questionnaire package that they completed and sent back to the Pain Research Lab at Queen's University. The questionnaire package contained a letter of information, consent form, postage-paid return envelope, the measures described below, as well as additional measures that are not part of the scope of the present study. Data from these participants were collected at baseline, six months, and at one year.

\section{Measures}

\section{Depressive symptoms}

Based on the symptoms of depression from the DSM-IV, the Patient Health Questionnaire 9 assessed depressive symptoms (PHQ-9) ${ }^{16}$ using a scale from 0 (Not at all) to 3 (Nearly every day) how often patients have experienced nine symptoms over the past two weeks (e.g., "Little interest or pleasure in doing things"). The PHQ-9 was chosen as it is a brief, validated measure of depressive symptoms in chronic pain patients. ${ }^{17} \mathrm{~A}$ severity score was calculated by summing all items that ranged from 0 (no depressive symptoms) to 27 (every depressive symptom nearly every day). Higher scores represent greater depressive symptoms. Cronbach's alpha for the PHQ-9 was good in this study $(\alpha=.90)$.

\section{Pain catastrophizing}

Catastrophizing was assessed using the widely used Pain Catastrophizing Scale (PCS) ${ }^{6}$ that consisted of 13 statements describing various thoughts and feelings regarding a patient's pain rated on a scale from 0 (Not at all) to 4 (All the time). The PCS is scored on three subscales: rumination (e.g., "I keep thinking about how much it hurts"), magnification (e.g., "I become afraid that the pain will get worse"), and helplessness (e.g., "It’s terrible and I think it’s never 
going to get any better"), or as a total score. The total score ranges from 0 to 52, with higher scores representing greater pain catastrophizing. Cronbach's alpha for total PCS was good ( $\alpha=$ $.96)$.

\subsubsection{Emotion regulation}

The Difficulties in Emotion Regulation Scale (DERS) ${ }^{18}$ consisted of 36 items concerning one's ability to manage emotions (e.g., "I experience my emotions as overwhelming and out of control"). The DERS is a comprehensive measure of difficulties in emotion regulation that assesses awareness and understanding of emotions, acceptance of emotions, ability to effectively use strategies to regulate emotion, and ability to control impulses and engage in goal directed behaviour when experiencing negative emotions. ${ }^{18}$ Patients indicate the degree to which they agree with each thought or feeling using a five-point scale from 1 (Almost never) to 5 (Almost always). The total score ranges from 36-180, with higher scores indicating more difficulties in emotion regulation. Cronbach's alpha was good for this study $(\alpha=.95)$.

\subsubsection{Pain}

Pain was assessed using the Short-Form McGill Pain Questionnaire (SF-MPQ), ${ }^{19}$ a measure recommended for use in chronic pain trials. ${ }^{20}$ Patients rated 15 pain related terms that describe their current pain as rated on a four-point scale ( $0=$ None, $3=$ Severe $)$. Eleven items describe sensory pain (e.g., "throbbing", "stabbing"), while four describe affective pain (e.g., "tiringexhausting”, "punishing-cruel”). A total pain score is calculated by summing all responses with higher scores representing greater pain, with scores ranging from 0 to 45 . For this study, Cronbach's alpha for the SF-MPQ was good $(\alpha=.92)$.

\section{Data analysis}

Statistical Package for the Social Sciences (SPSS, Version 22) was used to conduct all analyses. Missing data were estimated for cases with $80 \%$ or higher completion within each measure; cases with less than $80 \%$ completion for any measure were excluded.

The self-regulation models were tested using serial mediation, or model 6 of version 3.0 of Hayes ${ }^{, 21}$ PROCESS macro. Using a bootstrapping approach, it provides a statistical test for the indirect effect of the mediators and can be used to examine multiple mediation models. Confidence intervals for indirect effects are calculated by resampling with replacement from the original data thousands of times, estimating the model for each sub-sample, calculating the indirect effects themselves, and finding the endpoints of a confidence interval for each model. An indirect effect is significantly different from zero if its confidence interval does not contain zero. In both analyses, a bootstrap confidence interval was constructed using 10000 bootstrap samples.

Serial mediation assumes a causal flow from the independent variable, through both mediators, to the outcome. ${ }^{21}$ In this analysis, the independent variable was measured at time one, 
the two mediators were measured at time two (six months), and the outcome was measured at time three (one year). In each model, covariates were added in order to control for values of the mediators and outcome variables at baseline. Each analysis contains one direct effect and four indirect effects. The total direct effect is the relationship between the independent variable and the outcome, while removing the effect of the mediators. There are three specific indirect effects that are estimated as products of regression coefficients linking to the outcome. The fourth indirect effect, the total indirect effect, is the sum of all specific indirect effects. If measures with subscales are found to be significant, they will be followed by a subscale analysis examining the unique contribution of each subscale.

\section{Results}

\section{Model 1: Depression $\rightarrow$ Catastrophizing $\rightarrow$ Difficulties in emotion regulation $\rightarrow$ Pain}

The first model examined the path from depression at baseline, to catastrophizing at six months, to difficulties in emotion regulation at six months, to pain at one year. The total direct effect (i.e., the relationship between baseline depression and pain at one year, with the effect of the mediators removed) was not significant $(b=0.30, t(128)=1.91, p=.06)$. As Hayes has explained, the lack of a significant effect between baseline depression and pain at one year does not preclude a significant mediation. ${ }^{21}$ The total indirect effect (i.e., the sum of the specific indirect effects) was not significant ( $b=0.09, \mathrm{CI}=-.0057$ to .2463 ). The only significant specific indirect effect was baseline depression to catastrophizing at six months to pain at one year ( $b=$ 0.10 , CI $=.0049$ to .2520 ). Neither of the other indirect effects from baseline depression, through difficulties in emotion regulation at six months, to pain at one year $(b=-0.002, \mathrm{CI}=-.0542$ to 0.0284 ) or from baseline depression, through both catastrophizing and difficulties in emotion regulation at six months, to pain at one year $(b=-0.0006$, CI $=-.0150$ to .0284$)$ were not significant. Path coefficients can be seen in Figure 1. In summary, catastrophizing at six months mediated the relationship between baseline depression and pain at one year.

To follow up this significant finding, a mixed serial-parallel mediation was run, (model 80 of Hayes' PROCESS macro). This model examined the path from depression at baseline, to each component of catastrophizing (i.e., helplessness, magnification and rumination) at six months, to difficulties in emotion regulation at six months, to pain at one year (see Figure 2), while controlling for baseline catastrophizing, difficulties in emotion regulation, and pain. The total indirect effect was significant $(b=0.14, \mathrm{CI}=.0044$ to .3031$)$. The only significant specific indirect effect was baseline depression to helplessness at six months to pain at one year ( $b=$ 0.17 , CI $=.0282$ to .3826 ). In summary, helplessness was the driving factor of catastrophizing in the relationship between baseline depression and pain at one year. 


\section{Model 2: Pain $\rightarrow$ Catastrophizing $\rightarrow$ Difficulties in Emotion Regulation $\rightarrow$ Depression}

The second inverse model examined the path from baseline pain to catastrophizing at six months, to difficulties in emotion regulation at six months, to depression at one year (see Figure 1). The total direct effect (i.e., the relationship between baseline pain and depression at one year, excluding the effect of the mediators) was not significant $(b=0.04, t(128)=0.91, p=.36)$. The total indirect effect (i.e., the sum of the specific indirect effects) was also not significant ( $b=$ $0.001, \mathrm{CI}=-.0284$ to .0261 ). None of the indirect paths in this model were significant. These include the path from baseline pain through catastrophizing at six months, to depression at one year $(b=-0.002$, CI $=-.0196$ to .0033), the path from baseline pain through difficulties in emotion regulation at six months to pain at one year $(b=0.002$, CI $=-.0204$ to .0279$)$, and the path from baseline pain through both catastrophizing and difficulties in emotion regulation at six months, to depression at one year $(b=-0.001, \mathrm{CI}=-.0077$ to .0021$)$. In summary, catastrophizing or difficulties in emotion regulation did not explain the relationship between baseline pain and depression at one year.

\section{Discussion}

This study examined the relationship between pain and depression through patient catastrophizing and emotion regulation. Baseline depression leads to catastrophizing six months later, which leads to worse pain at one year. This occurs over and beyond levels of pain, catastrophizing, and difficulties in emotion regulation at baseline. Catastrophizing is an important variable in the relationship between depression and pain, suggesting that IC/BPS pain management strategies supports the reduction of elevated catastrophizing as previously recommended. ${ }^{22,23}$ These results also underscore how pain-specific catastrophizing, not general emotion regulation abilities, plays a role in the long-term relationship between depression and pain in women with IC/BPS. Specifically, it was the helplessness domain of pain catastrophizing, a patient's feelings of lost control over their pain, that explained the longitudinal relationship between depression and pain over one year. Tripp et al. ${ }^{24}$ have suggested that helplessness becomes more important in predicting patient outcomes as the time since diagnosis increases. With women in this sample diagnosed with IC/BPS for an average of eight and a half years, helplessness seems to be a salient concern in the patient's conceptualization of their disease and for its pain management. When experiencing pain, patients' make a primary appraisal of the pain that determines whether their pain is perceived as threatening. In a secondary appraisal process, the pain and the situation that the pain occurs in are then evaluated against the individual's belief that they can manage that pain (i.e., threat) using coping strategies they have likely used in the past. As well, the person examines their belief that this coping will lead to a successful reduction in the pain. In this secondary appraisal of pain, if the coping does not meet the threat level for that pain situation, or over a series of pain situations, then thoughts of helplessness with pain management are likely. In turn, these helplessness thoughts can generate greater anxious thinking when pain is present and thus reinforce greater helplessness unless this negative cycle is 
intervened upon. ${ }^{25,26,27}$ The findings from this study reinforce the importance of appropriate referral for therapeutic intervention addressing patient feelings of helplessness about their pain.

These results also show that baseline depression can lead to increased feelings of helplessness at six months, which in turn leads to greater pain at one-year. It is possible that a depressed mood may cast a negative light on one's perceived ability to cope with pain over time or it may reflect feelings of helplessness. There is evidence that depression level (high or low) moderates the effect of patient coping in the relationship between catastrophizing and pain. ${ }^{28}$ Thus, clinicians may consider appropriate antidepressant intervention alongside a referral to a psychosocial de-catastrophizing program to optimise pain management outcomes.

There are several limitations. First, although the majority of the women in this study were Caucasian, the prevalence of IC/BPS or the impact on quality of life does not change by race or ethnicity. ${ }^{29}$ However, this one-year analysis of a heterogeneous cohort of patients at different times in their personal IC/BPS clinical journey should be considered as only representative of the described pathways that they are predicted to likely follow. Secondly, it is unclear whether these results would generalize to men with IC/BPS. Thirdly, attrition created the case where the analyses used 135 women that completed all three time-points, losing power when compared to the larger sample collected. However, the results from the models were significant, indicating statistical and theoretical robustness of these models. Finally, these longitudinal analyses were conducted on women who have been living with IC/BPS for an average of eight and a half years. Therefore, these findings may not generalize well to newly diagnosed patients, as most women in this sample have had IC/BPS many years. Future research should investigate these models in newly diagnosed patients.

\section{Conclusion}

This study shows that catastrophizing, and specifically helplessness, has a key role in the maintenance of the negative relationship between depression and pain over time in IC/BPS. Thus, a more comprehensive and effective management of patients with longstanding IC/BPS must consider the significant impact of helplessness and depression on the patient pain experience. 


\section{References}

1. Nickel JC, Shoskes D, Irvine-Bird K. Clinical phenotyping of women with interstitial cystitis/painful bladder syndrome: a key to classification and potentially improved management. J Urol 2009; 182: 155-60.

2. Suskind AM, Berry SH, Suttorp MJ, et al. Symptom persistence in a community cohort of women with interstitial cystitis/bladder pain syndrome (IC/BPS): 3-, 6-, 9-, and 12month follow-up from the RICE cohort. Int Urogynecol J 2014; 25: 1639-43.

3. Clemens JQ, Brown SO, Calhoun EA. Mental health diagnoses in patients with interstitial Cystitis/Painful bladder syndrome and chronic Prostatitis/Chronic pelvic pain syndrome: A Case/Control study. J Urol 2008; 180: 1378-82.

4. Tripp DA, Nickel JC, Wong J, et al. Mapping of pain phenotypes in female patients with bladder pain syndrome/interstitial cystitis and controls. Eur Urol 2012; 62: 1188-94.

5. Naliboff BD, Stephens AJ, Lai HH, et al. Clinical and psychosocial predictors of urological chronic pelvic pain symptom change in 1 year: A prospective study from the MAPP Research Network. J Urol 2017; 198: 848-57.

6. Sullivan MJL, Bishop SR, Pivik J. The Pain Catastrophizing Scale: Development and validation. Psychol Assess 1995; 7: 524-32.

7. Geisser ME, Robinson ME, Keefe FJ, et al. Catastrophizing, depression and the sensory, affective and evaluative aspects of chronic pain. Pain 1994; 59: 79-83.

8. Linton SJ, Bergbom S. Understanding the link between depression and pain. Scand $J$ Pain 2011; 2: 47-54.

9. Williams AC, \& Shäfer, G. How do we understand depression in people with persistent pain? J Contemp Psychother 2016; 46: 149-57.

10. Nickel JC, Tripp DA, Pontari M, et al. Psychosocial phenotyping in women with interstitial cystitis/painful bladder syndrome: a case control study. J Urol 2010; 183: 16772.

11. Katz L, Tripp DA, Carr LK, et al. Understanding pain and coping in women with intersittial cystitis/bladder pain syndrome. BJU International 2017; 120: 286-92.

12. Tripp DA, Nickel JC, FitzGerald MP, et al. Sexual functioning, catastrophizing, depression, and pain, as predictors of quality of life in women with interstitial cystitis/painful bladder syndrome. Urology 2009; 73: 987-92.

13. Koechlin H, Coakley R, Schechter N, et al. The role of emotion regulation in chronic pain: A systematic literature review. J Psychosom Res 2018; 107: 38-45.

14. Linton SJ, Flink IK, Schrooten MGS, et al. Understanding co-occurring emotion and pain: The role of context sensitivity from a transdiagnostic perspective. J Contemp Psychother 2016; 46: 129-37. 
15. Trindade IA, Ferreira C, Pinto-Gouveia J. The longitudinal effects of emotion regulation on physical and psychological health: A latent growth analysis exploring the role of cognitive fusion in inflammatory bowel disease. Br J Health Psychol 2018; 23: 171-85.

16. Kroenke K, Spitzer RL, Williams JBW. The PHQ-9: Validity of a brief depression severity measure. J Gen Intern Med 2001; 16: 606-13.

17. Choi Y, Mayer TG, Williams MJ, et al. What is the best screening test for depression in chronic spinal pain patients? The Spine Journal 2014; 14: 1175-82.

18. Gratz KL, Roemer L. Multidimensional assessment of emotion regulation and dysregulation: development, factor structure, and initial validation of the Difficulties in Emotion Regulation Scale. J Psychopathol Behav Assess 2004; 26: 41-54.

19. Melzack R. The short-form McGill Pain Questionnaire. Pain 1987; 30: 191-97.

20. Dworkin RH, Turk DC, Farrar JT, et al. Core outcome measures for chronic pain clinical trials: IMMPACT recommendations. Pain 2005; 113: 9-19.

21. Hayes AF. Introduction to mediation, moderation, and conditional process anlaysis. Second ed. Methodology in social sciences, ed. T.D. Little. New York: Guilford Press. 2018.

22. Tripp DA. Managing psychosocial correlates of urologic chronic pelvic pain syndromes: Advice from a urology pain psychologist. Can Urol Assoc J 2018; 12: S175-S77.

23. Tripp DA, Nickel JC. The psychology of urologic chronic pelvic pain: A primer for urologists who want to know how to better manage chronic prostatitis and interstitial cystitis. AUA Update Ser 2011; 30: 385-96.

24. Tripp DA, Nickel JC, Ross S, et al. Prevalence, symptom impact and predictors of chronic prostatitis-like symptoms in Canadian males aged 16-19 years. BJU International 2009; 103: 1080-84.

25. Lazarus RS, Folkman S. Stress, Appraisal, and Coping. New York: Springer Pub. Co. 1984.

26. Sullivan MJ, Thorn B, Haythornthwaite JA, et al. Theoretical perspectives on the relation between catastrophizing and pain. Clin J Pain 2001; 17: 52-64.

27. Severeijns R, Vlaylen JWS, van den Hout MA. Do we need a communal coping model of pain catastrophizing? An alternative explanation. Pain 2004; 111: 226-29.

28. Muere A, Tripp DA, Nickel JC, et al. Depression and coping behaviors are key factors in understanding pain in interstitial cystitis/bladder pain syndrome. Pain Manag Nurs 2018.

29. Clemens JQ, Joyce GF, Wise M, et al., Interstitial Cystitis and Painful Bladder Syndrome, in Urologic Diseases in America; NIH Publication No. 07-5512, M.S. Litwin and C.S. Saigal, Editors. 2007, U.S. Government Publishing Office: Washington, DC. p. $124-54$. 


\section{Figures and Tables}

Fig. 1. Path coefficients of models. Note: Significant coefficients are in bold $\left({ }^{*} \mathrm{p} \leq 0.05\right)$. The significant indirect path is highlighted in green.

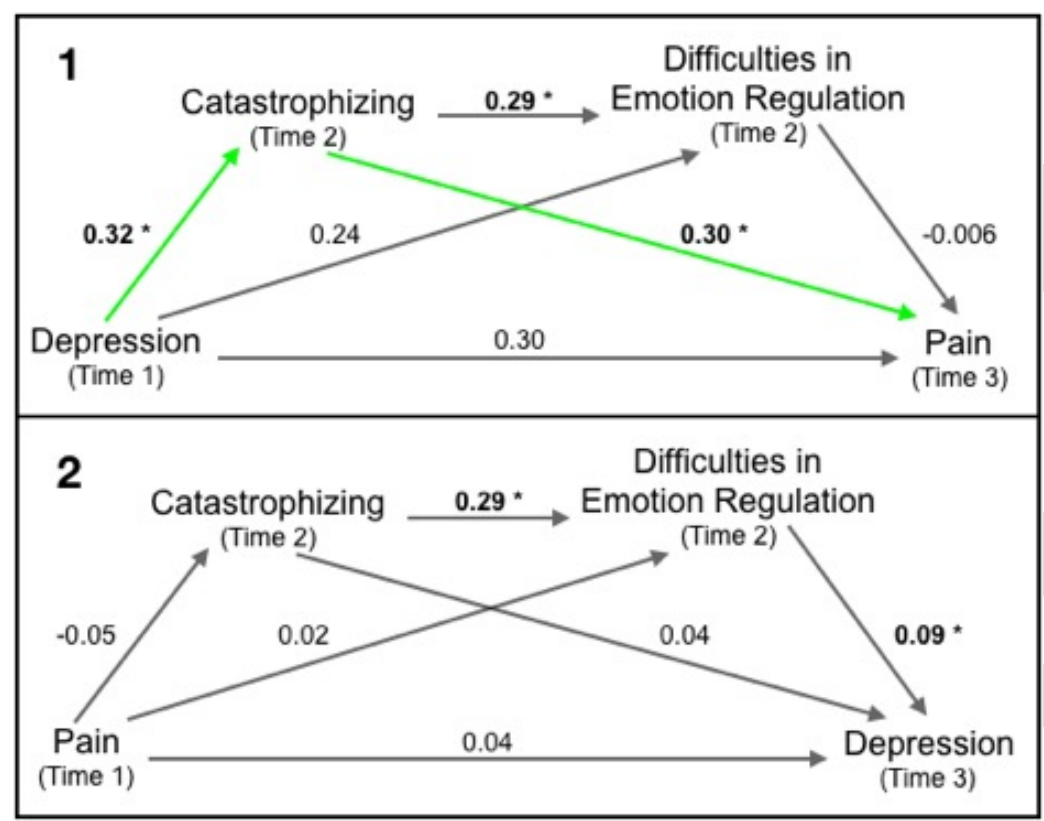

Fig. 2. Followup mediation of model 1. Note: In this model, pain, total catastrophizing, and difficulties in emotion regulation at baseline were included as covariates. Significant coefficients are in bold $\left({ }^{*} \mathrm{p} \leq 0.05\right)$. The significant indirect path is highlighted in green.

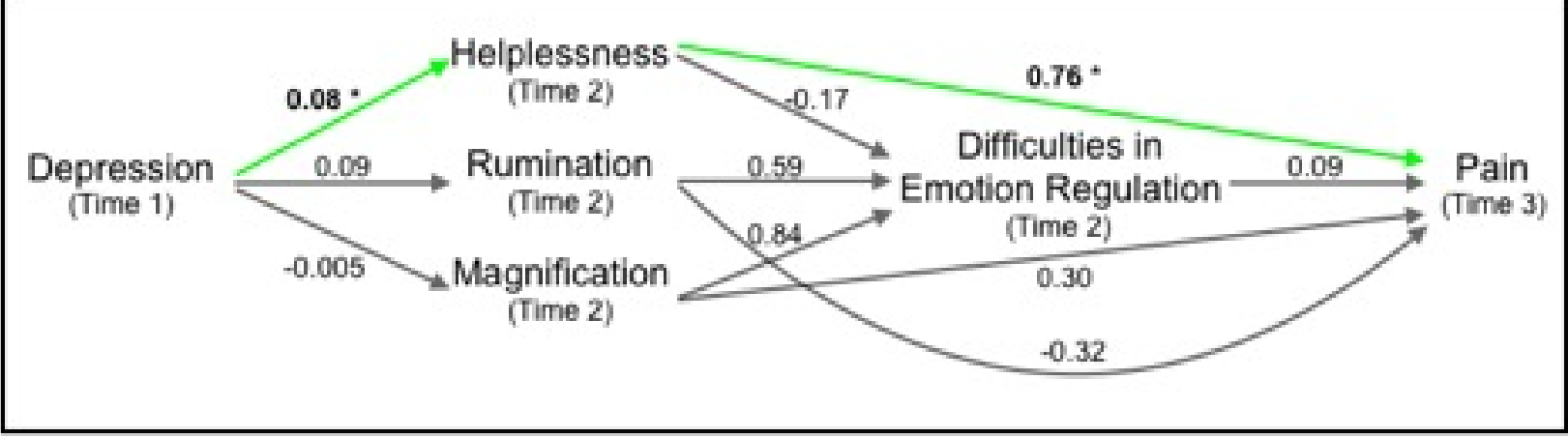




\begin{tabular}{|l|l|c|}
\hline Table 1. Demographic characteristics of the sample \\
\hline & & $\mathbf{n}(\%)$ \\
\hline Ethnicity & White & $133(98.5 \%)$ \\
& Black/African-Canadian & $1(0.7 \%)$ \\
& Hispanic/Latino & $0(0.0 \%)$ \\
& Aboriginal & $0(0.0 \%)$ \\
& Other & $1(0.7 \%)$ \\
& Missing & $0(0.0 \%)$ \\
\hline Education level & Less than high school & $3(2.2 \%)$ \\
& High school/GED & $22(16.3 \%)$ \\
& Some college/university & $28(20.7 \%)$ \\
& Graduate from college/university & $47(34.8 \%)$ \\
& Graduate/professional school after college/university & $35(25.9 \%)$ \\
& Missing & $0(0.0 \%)$ \\
\hline Living with spouse/partner & Yes & $83(61.5 \%)$ \\
& No & $51(37.8 \%)$ \\
& Missing & $1(0.7 \%)$ \\
\hline Employment status & Employed & $57(42.2 \%)$ \\
& Unemployed & $7(5.2 \%)$ \\
& Student & $3(2.2 \%)$ \\
& Retired & $41(30.4 \%)$ \\
& Disabled & $25(20.0 \%)$ \\
& Missing & $0(0.0 \%)$ \\
\hline
\end{tabular}

This table outlines the demographic characteristics of the participants that completed the questionnaires at baseline, six months, and one year. The total $n=135$. 


\begin{tabular}{|c|c|c|c|}
\hline & Baseline & 6 months & 1 year \\
\hline $\begin{array}{l}\text { Medications (e.g., antibiotics, opioids, non-opioids, } \\
\text { bladder instillations) }\end{array}$ & 130 (96.30\%) & 123 (91.11\%) & 129 (95.56\%) \\
\hline $\begin{array}{l}\text { Surgical interventions (e.g., cauterization, } \\
\text { hydrodistention, Botox) }\end{array}$ & 37 (27.41\%) & 35 (25.93\%) & 37 (27.41\%) \\
\hline $\begin{array}{l}\text { Physical manipulations (e.g., pelvic floor physiotherapy, } \\
\text { acupuncture) }\end{array}$ & 38 (28.15\%) & 44 (32.59\%) & 50 (37.04\%) \\
\hline $\begin{array}{l}\text { Psychological interventions (e.g., psychotherapy, } \\
\text { meditation, hypnosis) }\end{array}$ & $30(22.22 \%)$ & $30(22.22 \%)$ & $26(19.26 \%)$ \\
\hline $\begin{array}{l}\text { Alternative treatments (e.g., herbal supplements, } \\
\text { naturopathy, biofeedback, homeopathy) }\end{array}$ & $37(27.41 \%)$ & $35(25.93 \%)$ & $41(30.37 \%)$ \\
\hline Diet related interventions & $8(5.93 \%)$ & $13(9.63 \%)$ & $6(4.44 \%)$ \\
\hline
\end{tabular}

This table summarizes the types of treatments the patients received throughout the study. 